\title{
Correction to: Factors Associated with Accurate Analysis of Fischer-Tropsch Products
}

\author{
Kang Xiao ${ }^{1} \cdot$ Xingzhen $\mathrm{Qi}^{2} \cdot$ Xinxing Wang ${ }^{2} \cdot$ Dong $\mathrm{Lv}^{2} \cdot$ Mingxiong Zhu ${ }^{3} \cdot$ Liangshu Zhong $^{2}$
}

Published online: 14 July 2018

○) Springer Science+Business Media, LLC, part of Springer Nature 2018

Correction to: Catalysis Letters (2017) 147:704-715

https://doi.org/10.1007/s10562-016-1958-4

The authors regret that a certain error in their manuscript was not find in time during manuscript proof, which might puzzle readers. The corrected section now appear here.

In Section 2.4 Products Selectivity, page 710 line 1 (exclude figures and tables), the equation $\sum_{i} S_{1}^{i} \equiv 100 \%$ should be $\sum_{i} S_{1}^{i} \not \equiv 100 \%$

since "The sum of selectivity of all collected products by $S_{1}$ $\left(S_{1}{ }^{i}\right)$ might not be $100 \%$ due to accumulated or lost products or extra ones." (page 709, the last sentence).

The original article can be found online at https://doi.org/10.1007/ s10562-016-1958-4.

Liangshu Zhong

zhongls@sari.ac.cn

1 School of Materials Science \& Engineering, Nanjing

University of Posts and Telecommunications,

Nanjing 210023, People's Republic of China

2 CAS Key Laboratory of Low-Carbon Conversion Science and Engineering, Shanghai Advanced Research Institute, Chinese Academy of Sciences, Shanghai 201210, People's Republic of China

3 Jiulongpo Environmental Protection Bureau of Chongqing, Chongqing 400050, People's Republic of China 